\title{
Extracción de aceite de aguacate: Un experimento industrial
}

\author{
Por L. Martínez Nieto* (1), R. Barranco Barranco (2) \\ y M. V. Moreno Romero (1). \\ (1) Departamento de Ingeniería Química. Universidad de Granada. Campus Universitario \\ de Fuentenueva s/n. 18071 - GRANADA. \\ (2) Destilaciones García de la Fuente. S.A. (D.G.F.). GRANADA.
}

\section{RESUMEN}

\section{Extracción de aceite de aguacate: Un experimento industrial.}

Se analizan los resultados obtenidos en un experimento industrial de obtención de aceite de aguacate por un proceso continuo similar al de obtención de aceite de oliva, indicando las posibles modificaciones para mejorar el rendimiento.

PALABRAS-CLAVE: Aceite de aguacate (obtención) - Experimento industrial - Proceso continuo.

\section{SUMMARY}

Avocado oil extraction: An industrial experiment.

The results of an industrial experiment to obtain avocado oil by continuous process similar to that for obtaining olive oil are analysed. Possible ways of improving the method and thus the yield are pointed out.

KEY-WORDS: Avocado oil (obtaining) - Continuous process - Indus trial experiment.

\section{INTRODUCCION}

La producción de aguacates en la zona del litoral mediterráneo está alcanzando niveles que se aproximan a las $100.000 \mathrm{Tm}$ que en la actualidad se destinan, en su integridad, al mercado exterior y al consumo interno. La superficie destinada al cultivo aumenta año tras año no aumentando de la misma manera la demanda para el consumo por lo que cabe esperar a corto plazo un excedente importante. Si a este excedente se le une la fruta rechazada en los canales de exportación así como el "destrio" y la fruta de segunda floración inmadura, fruta caída del árbol, etc, pueden resultar cantidades apreciables a la que es necesario buscarle una aplicación.

Algunos autores han puesto de manifiesto las excelencias del aceite de aguacate en su aplicación cosmética, dermatológica y terapeútica (1), (2), (3), (4), (5), (6). Se encuentra abundante bibliografía en la extracción de aceite de aguacate a escala de laboratorio, generalmen- te por uso de disolventes (7), (8), (9), (10), (11), (12), pero son pocas las referencias a otros procedimientos de extracción que se hayan llevado a escala industrial.

En este sentido caben destacar las publicaciones de HAENDLER (13), que compara los rendimientos de la extracción por disolventes previo secado $(95 \%)$ con el obtenido por presión de la pulpa seca (80-85\%). SADIR (14), extrae con disolventes tras una fermentación anaeróbia de la pulpa, para facilitar la rotura celular obteniendo rendimientos próximos al 90\%. JAUBERT (15), obtiene rendimientos próximos al $80 \%$ por presión y sucesiva filtración, el mismo autor (16), propone un método de extracción por presión, decantación y posterior centrifugación de la fase oleosa.

TURATI (17), compara los rendimientos obtenidos por extracción con disolventes con pulpa secada (73\%) o liofilizada (85\%), con los obtenidos por presión empleando como coadyuvante cáscara de soja a 350 atm (73\%) y los obtenidos por centrifugación con adición de sal en el que se obtiene un rendimiento más bajo (55\%).

Desde el punto de vista de la maquinaria industrial propiamente dicha, se encuentra poca bibliografía. LANZANI (18), utiliza un sistema continuo PIERALISIS para extracción de aguacate deshuesado y con piel, que previa laminación y adición de agua se mantiene en batidora en caliente con ajuste de $\mathrm{pH}$. Posteriormente se manda a un separador de fases centrifugo para obtener el aceite con el agua y la pulpa húmeda. A continuación se centrifuga la mezcla aceite-agua como es usual. Partiendo de masa batida con el $20 \%$ de agua adicional se obtienen los mejores resultados a $60^{\circ} \mathrm{C}$ y 30 minutos de batido ajustando el $\mathrm{pH}$ a 4 , quedando una pasta con el $57-60 \%$ de humedad y obteniéndose un aceite de buena calidad con un 0 ' $3 \%$ de acidez, índice de peróxidos 7 ' 3 y con un rendimiento del $76 \%$ respecto de la cantidad total de aceite extraible por hexano.

En el presente trabajo se da cuenta de un experimento realizado a escala industrial con mezcla de aguacates de distintas variedades y tamaños procedentes de la zona litoral granadina utilizando un proceso continuo por centrifugación. Se ha utilizado el mismo equipo y se- 
cuencia que para la obtención de aceite de oliva y se llevó a cabo en Fuente Cardona S.A. de Ubeda (Jaén), con maquinaria propia y con maquinaria de la casa Westfalia Separator A.G.

\section{MATERIALES Y METODOS}

\subsection{Materia prima.}

Se ha partido de una mezcla de aguacates variedades "Fuerte», "Reed", "Hass" y "Antillana» en estado de maduración aceptable, dada la fecha (marzo 1989) en que se realizó el experimento. Por ser una fecha muy avanzada no se obtuvo aguacate "Bacon». En la Tabla I se encuentra la composición de la muestra utilizada.

Tabla I

Composición de la muestra utilizada.

\begin{tabular}{lrc} 
Variedad & $\mathrm{Kg} \cdot$ & \% en la muestra \\
\cline { 2 - 3 } Fuerte & 33 & $3^{\prime} 67$ \\
Hass & 459 & $51^{\prime} 00$ \\
Reed & 177 & $19^{\prime} 67$ \\
Antillana & 231 & $25^{\prime} 66$
\end{tabular}

Antes de proceder a la extracción industrial se procedió al análisis en el laboratorio de la riqueza grasa de la pulpa, previa desecación en horno microondas y extracción en soxhlet (12), y características de cada una de las variedades empleadas, índice de acidez (19), índice de peróxidos (20), absorbancia al U.V. $\mathrm{K}_{270}$ (21), (22), índice de refracción (23) y materia insaponificable (24). En la Tabla II se encuentra un resumen de las mismas. En la Tabla III se encuentra la composición en ácidos grasos obtenida por cromatografía gaseosa de los ésteres metílicos (25), (26) y en la Tabla IV la composición de la fracción esterólica del insaponificable (24), así como el análisis del eritrodiol (27).

\subsection{Proceso.}

Se ha seguido un proceso continuo con el equipo y secuencia similar al utilizado para la obtención de aceite de oliva cuyo esquema se puede asimilar al representado en la Figura 1. Las características de cada aparato son las siguientes:

- Molino triturador Fuentes Cardona (F.C.) 25-25 C.V.

- Termobatidora horizontal F.C. 2.000 Kg. -5'5 C.V.

- Bomba de trasiego 2 C.V.

- Decantador centrífugo horizontal Westfalia R.C.A. 450 - 30 C.V.

- Tamiz vibratorio 0'5 C.V.

- Separadores centrífugos verticales Westfalia S A 12 -7'5 C.V.

Tabla II

Riqueza grasa de la pulpa y carácteres analíticos del aceite en el laboratorio.

\begin{tabular}{|c|c|c|c|c|c|c|c|}
\hline \multirow[b]{2}{*}{ Variedad } & \multicolumn{2}{|c|}{ Aguacate } & \multicolumn{5}{|c|}{ Caracteres del Aceite } \\
\hline & \%Aceite & \% Agua & $\begin{array}{l}\text { I. Acidez } \\
(0) \\
\end{array}$ & $\begin{array}{l}\text { I.Peróxidos } \\
\left.\begin{array}{l}(\mathrm{mqO} \\
\end{array} 2 / \mathrm{Kg}\right) \\
\end{array}$ & $\begin{array}{c}\text { Absorbancia }\left(E^{\prime} 1 \mathrm{~cm}\right) \\
\text { U.V. }(270 \mathrm{~nm}) \\
\end{array}$ & $\begin{array}{c}\text { I. Refracción } \\
(20 \cong) \\
\end{array}$ & $\begin{array}{l}\text { Insaponificable } \\
(\%)\end{array}$ \\
\hline Fuerte & $26^{\prime} 81$ & $60 \cdot 92$ & 0.26 & $15^{\prime} 3$ & $0 \cdot 50$ & $1 \cdot 4705$ & $2 \cdot 59$ \\
\hline Hass & $22 \cdot 99$ & $66 \cdot 98$ & 0.65 & $15^{\prime} 0$ & 0.78 & $1 ' 4698$ & $2 \cdot 23$ \\
\hline Reed & $12 \cdot 81$ & 76.69 & 0.66 & $10^{\prime} 4$ & $1: 45$ & $1 \cdot 4705$ & $5 \cdot 34$ \\
\hline Antillana & $10 \cdot 73$ & $76 \cdot 61$ & $2 \cdot 60$ & $7 \cdot 2$ & $2 \cdot 05$ & $1 \cdot 4701$ & $2 \cdot 12$ \\
\hline
\end{tabular}

Tabla III

Composición en ácidos grasos.

\begin{tabular}{|c|c|c|c|c|c|c|c|c|c|c|}
\hline Variedad & $\% \mathrm{C} 14: 0$ & $\% \mathrm{C} 16: 0$ & $\%$ C $16: 1$ & $\% \mathrm{C} 17: 0$ & $\% \mathrm{C} 17: 1$ & $\% \mathrm{C} 18: 0$ & $\% \mathrm{C} 18: 1$ & $\% \mathrm{C} 18: 2$ & $\% \mathrm{C} 18: 3$ & $\% \mathrm{C} 20: 0$ \\
\hline Fuerte & 0.05 & $10 \cdot 75$ & $3 \cdot 14$ & 0.02 & 0.15 & 0.48 & $74 \cdot 32$ & $10^{\prime} 03$ & 0.85 & 0.05 \\
\hline Hass & 0.06 & $18 \cdot 62$ & $8 \cdot 47$ & --- & $0: 12$ & 0.49 & 60117 & $10 \cdot 97$ & 0.98 & 0.03 \\
\hline Reed & 0.06 & $18 \cdot 18$ & $6 \cdot 56$ & Trazas & 0,12 & 0.40 & $60 \cdot 25$ & $13^{\prime} 03$ & $1 \cdot 40$ & Trazas \\
\hline Antillana & 0.05 & $18 \cdot 87$ & $4 \cdot 16$ & --- & 0.15 & 0.59 & $63^{\prime} 07$ & $11^{\prime} 83$ & $1 \cdot 32$ & 0.08 \\
\hline
\end{tabular}

Tabla IV

Composición de la fracción esterólica del insaponificable.

Variedad
Fuerte
Hass
Reed
Antillana

\% Colesterol
No se detecta
Trazas
No se detecta
No se detecta

\% Campesterol
6'4
$4 \cdot 7$
8'6
$10 \cdot 6$

\begin{tabular}{c}
$\% \beta-$ sitosterol \\
\hline $92 ' 9$ \\
$95 ' 2$ \\
$91 ' 2$ \\
$89 \cdot 3$
\end{tabular}

\% Eritrodiol
No se detecta
No se detecta
No se detecta
Trazas




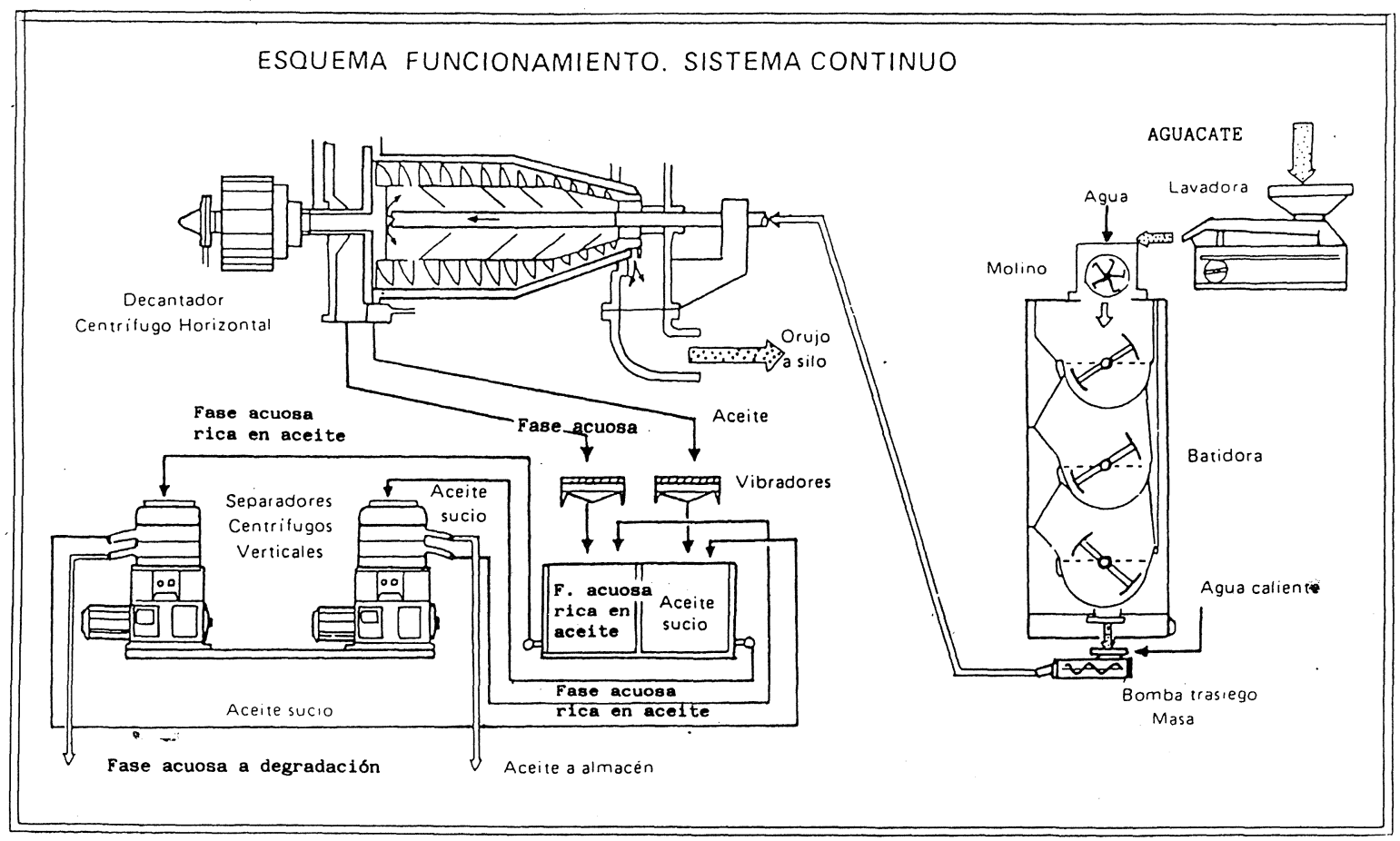

Figura 1

Obtención de aceite de aguacate a escala industrial. Esquema del proceso.

Se ha utilizado a diferencia de lo encontrado en bibliografía el aguacate íntegro, es decir, con piel y hueso. Dadas las características de la piel, fibrosa y de la pulpa, pastosa, para evitar atoramientos en la criba del molino de martillos se procedió a una molienda en húmedo agregando un $30 \%$ de agua en continuo sobre la cantidad de aguacates a molturar con lo que se ha comprobado que la molienda no plantea problemas.

La masa molida se mantuvo durante una hora en la batidora a $50^{\circ} \mathrm{C}$. Adicionada con un $70 \%$ de agua caliente sobre la masa de aguacates se trasvasó al decantador, de allí previo paso por los tamices vibradores se llevó a los separadores centrífugos verticales obteniéndose por último la separación del aceite y el agua.

En cada uno de los pasos anteriores se tomaron muestras para su posterior análisis. En el caso de la muestra de la batidora se tomó antes de agregar el agua para fluidizar la alimentación al decantador centrífugo. En la Tabla $V$ se encuentran los resultados para cada una de las muestras, pasta (con un $30 \%$ de agua), de fase acuosa, orujo de aguacate y aceite de proceso total.

\section{RESULTADOS Y DISCUSION}

En la Figura 2 se ha esquematizado el proceso de forma que permita realizar un balance de materia que conduzca a la obtención del rendimiento global en aceite.

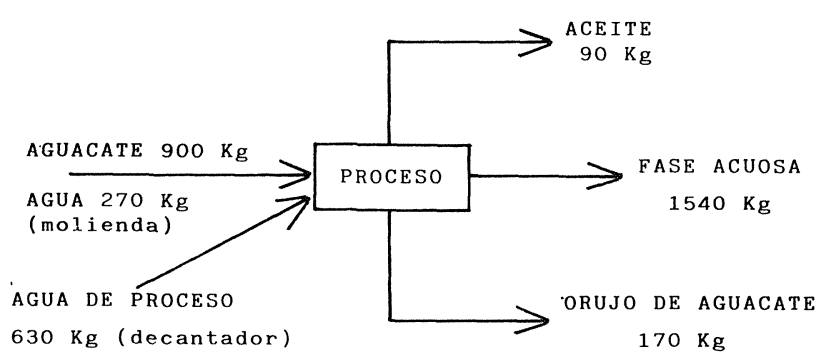

Figura 2

Flujos de entrada y salida. Balance de materia.

De los resultados de la Tabla $V$ para cada una de las corrientes de la Figura 2 se pueden deducir los siguientes parámetros para el proceso:

\begin{tabular}{lc} 
Corriente & $\%$ Aceite \\
\hline Pasta & $11^{\prime} 68$ \\
Fase acuosa & $2^{\prime} 80$ \\
Orujo de aguacate & $2^{\prime} 92$ \\
Aceite & 98,42
\end{tabular}

Tabla V

\begin{tabular}{|c|c|c|}
\hline$\%$ Agua & \% Sólidos & Tota? \\
\hline $71 \cdot 54$ & $16 \cdot 61$ & $99 \cdot 83$ \\
\hline $87 \cdot 13$ & $10^{\prime} 05$ & $99 \cdot 98$ \\
\hline $73 \cdot 25$ & $22 \cdot 85$ & $99 \cdot 02$ \\
\hline 0.75 & 0.83 & $100^{\prime} 00$ \\
\hline
\end{tabular}


A) Rendimiento neto en aceite:

$R=\frac{\text { Aceite obtenido }}{\text { Aceite en pasta extraíble con hexano }} \times 100$
$R=\frac{90 \times 0^{\prime} 9842}{1170 \times 0^{\prime} 1168} \times 100=64^{\prime} 81 \%$

B) Aceite en fase acuosa:

$1540 \mathrm{Kg} \times 0^{\prime} 028=43^{\prime} 12 \mathrm{Kg}$

C) Porcentaje de aceite en fase acuosa respecto al aceite de entrada:

$43^{\prime} 12$

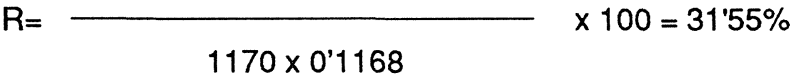

D) Aceite en el orujo de aguacate:

$170 \mathrm{Kg} \times 0$ 0'029 = 4'96 Kg

E) Porcentaje de aceite en el orujo de aguacates respecto al aceite de entrada:

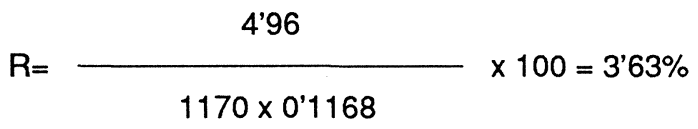

De estos resultados se puede deducir que el rendimiento neto en aceite es bajo, en comparación con los rendimientos obtenidos para el mismo proceso con aceituna, para obtención de aceite de oliva. Esto se puede justificar teniendo en cuenta que en el decantador no se hizo modificación ninguna, al objeto de tener en cuenta las diferentes peculiaridades que existen entre la pasta de aceituna y la de aguacate. Ello conduce sin duda a una buena separación de aceite del orujo de aguacate, pero no a una buena separación de las fases líquidas. La fase acuosa arrastra el $10^{\prime} 05 \%$ de sólidos y el aceite el 0'83\%, quizás debido al excesivo tiempo de molienda y al tipo de "pasta" que produce el aguacate.

Sin duda un acondicionamiento en el decantador en lo que se refiere a los anillos de separación y el uso de las cribas con diámetro superior al utilizado en la aceituna conduciría a una mejora en la separación de las fases, que redundaría en la obtención de una fase aceite más rica y mayor rendimiento, obteniéndose una fase acuosa con menor cantidad de aceite.

El aceite obtenido en el proceso se dejó reposar para la decantación de los sólidos, se filtró y se sometió a un proceso de decoloración usual, en cuanto a temperatura y porcentaje de tierra utilizada, en el Instituto de la Grasa y sus Derivados en Sevilla, con objeto de estudiar la influencia de la decoloración en la aplicabilidad cosmética del mismo.

De ambos aceites, antes y después de la decoloración, se hicieron análisis de los parámetros más usuales. En la Tabla VI se encuentran algunos de ellos. En la Tabla VII la composición de ácidos grasos obtenida por cromatografía gaseosa de los ésteres metílicos y en la Tabla VIII la composición de la fracción esterólica del insaponificable así como el eritrodiol.

Tabla VI

Carácteres analíticos del aceite obtenido.

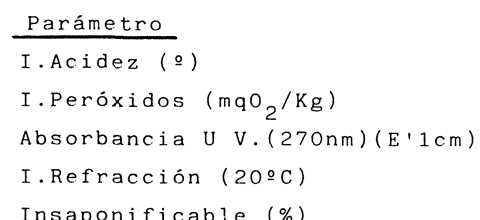

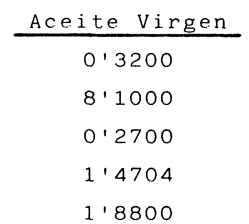

Tabla VII

Composición en ácidos grasos del aceite obtenido.

\begin{tabular}{|c|c|c|c|c|c|c|c|c|c|c|}
\hline Aceite & $\% \mathrm{C} 14: 0$ & $\% \mathrm{C} 16: 0$ & $\% \mathrm{C} 16: 1$ & $\% \mathrm{C} 17: 0$ & $\% \mathrm{C} 17: 1$ & $\% \mathrm{C} 18: 0$ & $\% \mathrm{C} 18: 1$ & $\% \mathrm{C} 18: 2$ & $\%$ C $18: 3$ & $\% \mathrm{C} 20: 0$ \\
\hline Virgen & 0.03 & $16 \cdot 55$ & $7 \cdot 17$ & 0.40 & 0.11 & 0,48 & $63 \cdot 83$ & $10 \cdot 93$ & $11^{\prime} 01$ & 0.02 \\
\hline Decolorado & 0103 & $16 \cdot 71$ & $6 \cdot 83$ & --- & 0,12 & 0.53 & $64 \cdot 01$ & $10 \cdot 67$ & 0.94 & 0.05 \\
\hline
\end{tabular}

Tabla VIII

Composición de la fracción esterólica del insaponificable del aceite obtenido.

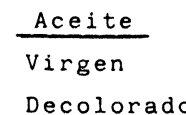

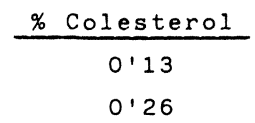

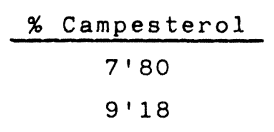

$9 \cdot 18$

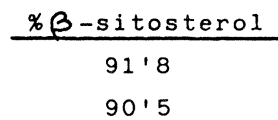


De la observación de dichas Tablas se deduce un ligero aumento de acidez, de índice de peróxidos y de absorbancia al U.V. $\mathrm{K}_{270}$, así como de insaponificable.

Cabe destacar, por lo extraño, la aparición en el aceite obtenido de un porcentaje significativo de eritrodiol, que no estaba presente en los aceites obtenidos de cada variedad, como se observa en la Tabla IV en la que sólo se encuentran, trazas en la variedad "Antillana" y para lo que no se encuentra justificación. No obstante la presencia de eritrodiol en aceites vírgenes de oliva de distintas procedencias incluso en valores superiores a lo permitido por la legislación (28) ya se ha puesto de manifiesto por distintos autores (29).

\section{CONCLUSIONES}

Del experimento de extracción industrial de aceite de aguacate llevado a cabo se puede concluir que: con la utilización del mismo proceso de extracción de aceite de oliva, con modificaciones puntuales en la fase molienda y en el decantador se puede obtener un buen rendimiento neto en aceite. Los parámetros de calidad son buenos tanto para el aceite virgen como para el decolorado.

\section{AGRADECIMIENTOS}

- A Destilaciones Garcia de la Fuente por el suministro del aguacate extraído.

- A Fuentes Cardona y Westfalia por la utilización de su maquinaria.

- Al Instituto de la Grasa y sus Derivados por su desinteresada colaboración en la decoloración del aceite en sus plantas piloto.

\section{BIBLIOGRAFIA}

1. Dupaigne, $P$. «Une nouvelle specialite pharmaceutique: L'insaponifiable de l'huile d'avocat".- Fruits 25 (1970) 915-916.

2. Daniel, A. - "Action des insaponifiables d'avocat et de soja sur l'inflamation du tissu conjontif".- Extrait de la "Gazette Medicale de France" 86 (1979) 2461-2468.

3. Chanez, J.F., Lamaud, E. Wepierre, J. - Activite biologique des insaponifiables d'huiles d'avocat et de soja. Interêt en cosmetologie".Laboratoire de pharmacologie, Center d'Estudes Pharmaceutiques. Univerté París XI 92290 Chatenay Malabry.
4. Hincky, M. -mInterêt en dermatologie d'un oleat d'insaponifiables d'huiles d'avocat et de soja".- Extrait de la "Gazette Medicale de France" 83 (1976) 1171-1172.

5. Thiers, M.H. -"Les insaponifiables d'huiles de soja et d'avocat Traitement de certaines modalités de la doleur artrosique".- Le Journal de Medicine de Lyon (1972) 5 Febrero 195-198.

6. Thiers, M.H. - L'huile d'avocat et son insaponifiable en cosmetologie et en therapeutique dermatologique ou medicale".- Fruits 26 (1971) 133-136.

7. Gómez, M.E. y Mora, C.E. -»Extracción de aceite de aguacate».Rev. Ion Bucaramanga (Colombia) 6 (1982) 91-97.

8. Alter, M. y Gutfinger, T. - Phospholopids in several vegetable oils”.Riv. Ital, Sostanze Grasse 59 (1982) 14-18.

9. Lewis, C.E., Morris, R., O'brien, K. -»The oil content of avocado mesocarp".- J. Sci. Food Agric. 29 (1978) 943-949.

10. Tango, J.S., da Costa, S.I., Antunes, A.J., Figuiredo, I.B. - COmposition du fruit et de l'huile de différentes variétès d'avocats cultivès dans l'etat de Sao Paulo".- Fruits 27 (1972) 143-146.

11. Brian, I.B. - - Isolation of umpleasant flavor compounds in theavocadon.J. Agr. Food Chem. 20 (1972) 753-757.

12. Martinez Nieto, L., Camacho Rubio, F., Rodriguez Vives, S., Moreno Romero, $M^{a}$ V. -„Extracción y caracterización del aceite de aguacate".- Grasas y Aceites 39 (1988) 272-277.

13. Haendler, L. $-m L$ 'huile d'avocat et les produits dérivès du fruit».- Fruits 20 (1965) 625-633.

14. Sadir, R. - Olio di abacate: Tecnologia de la raffinazione.»- Riv. Ital. Sostanze Grasse 49 (1972) 117-120.

15. Jaubert, J.N. - Une nouvelle techniche de préparation et da raffinage de l'huile d'avocat".- Fruits 25 (1970).

16. Jaubert, J.N. -"L'huile d'avocat: propiétès technologie".- Industr. Alim. (1970) 1411-1416.

17. Turati, J.M., Santos, L.C., Tango, J.S., Marina, H.K. -"Caracterizaçáo do óleo de abacate obtido por diferentes procesos de extraçaó".- Bol Ital. Campinas 22 (1985) 267-284.

18. Lanzani, A., Bondioli, P., Mariani, C., Fedeli, E., Ponzetti, A., Pieralisi, G. - - Tecnologia integrale di estrazione della sostanza grassa e di preparazione di farine dal frutto di avocado".- Riv. Ital. Sostanze Grasse 63 (1986) 487-492.

19. Norma U.N.E. 55011

20. Norma U.N.E. 55023.

21. Wolf, J.P. - Etude spectrophotometrique du rancisement des huiles".Rev. Fr. Corps Gras 1 (1954) 214-226.

22. Norma Consejo Oleicola Internacional (1957)

3. Norma U.N.E. 55015

24. Norma U.N.E. 55019.

25. Norma U.N.E. 55037.

26. Métodos de Análisis de Aceites y Grasas. Ministerio de Agricultura $n^{\circ}$ 41.

27. Norma U.N.E. 55136.

28. Real Decreto 259/1985 20 - Febrero.

29. Albi, T., Lanzón, A., Cert, A. y Aparicio, R. -„Valores de eritrodiol en muestras de aceite de oliva vírgenes andaluces".- Grasas y Aceites 41 (1990) 167-170.

(Recibido: Abril 1991) 